\title{
Synchronization control of stochastic delayed Lotka-Volterra systems with hardware simulation
}

\author{
Lan Wang ${ }^{1,2}$, Yiping Dong ${ }^{3 *}$, Da Xie ${ }^{3}$ and Hao Zhang ${ }^{4}$
}

"Correspondence:

yp.dong@foxmail.com

${ }^{3}$ China Key System \& Integrated

Circuit Co., Ldt., Wuxi, P.R. China

Full list of author information is

available at the end of the article

\begin{abstract}
In this paper, the synchronization control of a non-autonomous Lotka-Volterra system with time delay and stochastic effects is studied. The purpose is to firstly establish sufficient conditions for the existence of global positive solution by constructing a suitable Lyapunov function. Some synchronization criteria are then derived by designing an appropriate full controller and a pinning controller, respectively. Finally, an example is presented to illustrate the feasibility and validity of the main theoretical results based on the Field-Programmable Gate Array hardware simulation tool.
\end{abstract}

Keywords: Synchronization control; Lotka-Volterra system; Stochastic effects; Lyapunov function; Field-Programmable Gate Array

\section{Introduction}

The Lotka-Voltera (LV) system is one of the famous biological models which describe the interaction of a predator-prey model and consist of nonlinear ordinary differential equations, developed by Lotka and Volterra [1]. Over the past 20 years, the LV system has been extensively investigated from different aspects such as stability, control, evolutionary dynamics [2-7]. Reference [2] investigated the existence of a spatially steady state solution by applying a Lyapunov-Schmidt reduction. Based on many natural predator-prey interactions which sometimes exhibit stability, Ref. [3] checked the effect of small immigration and the inclusion of a nonlinear interaction term to the stability of the LV system. Lyapunov stability theory has been used to investigate the attractivity and synchronization of the LV system with mutual interference and with unknown parameters $[4,5]$. The global dynamics of a classical LV system was investigated with the effects of competition ability and impulsive periodic disturbance in Refs. [6, 7]. Besides, time delays are ubiquitous in the processing of information transmission because of the limited resource and environmental disturbance, which may cause undesired dynamics like oscillation, bifurcation and instability. Until now, the dynamical problem of a hybrid system with time delays has become a significant issue [8-11]. Moreover, a classical stochastic delayed LV model can be sharing, adaptation, distribution and reproduction in any medium or format, as long as you give appropriate credit to the original author(s) and the source, provide a link to the Creative Commons licence, and indicate if changes were made. The images or other third party material in this article are included in the article's Creative Commons licence, unless indicated otherwise in a credit line to the material. If material is not included in the article's Creative Commons licence and your intended use is not permitted by statutory regulation or exceeds the permitted use, you will need to obtain permission directly from the copyright holder. To view a copy of this licence, visit http://creativecommons.org/licenses/by/4.0/. 
described as follows [11]:

$$
\mathrm{d} x_{i}(t)=x_{i}(t)\left[r_{i}-\sum_{j=1}^{n} a_{i j} x_{j}(t)+\sum_{j=1}^{n} b_{i j} x_{j}(t-\tau)\right] \mathrm{d} t+\sigma_{i}(t) x_{i}(t) \mathrm{d} \omega_{i}(t),
$$

where $x_{i}(t)(>0)$ denotes the density of the $i$ th species at time $t, \omega_{i}(t), 1 \leq i \leq n$ are independent standard Brownian motions, and $r_{i}, a_{i j}, b_{i j}, \sigma_{i}$ are nonnegative. In mathematical ecology, Eq. (1) describes an $n$-species predator-prey system in which individuals compete with each other.

Some related work on the dynamical of predator-prey system has been presented based on the model (1). In Ref. [11], a global positive solution, stochastic permanence and extinction were discussed. In Ref. [12], the sufficient conditions for the existence of global positive solutions were obtained based on the LV model with random perturbation. Sufficient and necessary conditions were established for persistence and extinction of the systems under the condition of Lévy noise [13]. Reference [14] studied the positivity and the global stability of nontrivial solutions based on the Euler-Maruyama scheme for a twodimensional model of stochastic predator-prey interactions. Reference [15] discussed a novel LV system with infinite delay and feedback control through Lyapunov functionals and a new analysis technique.

Up till now, a great deal of research effort has focused on the LV model (such as Refs. [1619]). To the best of our knowledge, the synchronization control problems for the stochastic LV system have not yet been studied much. In fact, synchronization control problems can be found in almost everywhere in the real world. For example, in order to protect endangered species, we can design a reasonable optimal control strategy and adopt synchronous control to achieve effective animal protection. As is well known, a linear feedback control technique was used to control the LV model, and the Lyapunov direct method is used to synchronize two models [20]. In Ref. [21], a predator-prey model was established with Allee effect and seasonally forcing, and was controlled to follow a reference model by a synchronization approach.

However, it still remains a big challenge to deal with the LV system with delay and stochasticity. In the past few years, the synchronization control problems for complex networks have drawn much attention of researchers on the neural network, genetic regulatory networks and social networks [22-25]. This helps us to study the synchronization control problem for population systems based on LV models. At the same time, we will present a calculation example based on the Field-Programmable Gate Array (FPGA) hardware tool, which can work with the data faster and more efficiently.

In this paper, we study a stochastic delayed LV system with a control term. The structure of this paper is as follows. The model description and preliminaries are formally presented in Sect. 2. Some synchronization criteria are derived by the full controller and pinning controller, respectively, in Sect. 3. An example is presented to demonstrate the validity of the proposed methods based on the FPGA hardware tool which can deal with the data faster and more efficiently in Sect. 4. Finally, the conclusion is drawn in Sect. 5.

Notations: Throughout this letter, let $\left(\Omega, \mathscr{F},\left\{\mathscr{F}_{t}\right\}_{t \geq 0}, P\right)$ be a complete probability space with a filtration $\left\{\mathscr{F}_{t}\right\}_{t \geq 0}$ satisfying the usual conditions (i.e., it is increasing and right continuous while $\mathscr{F}_{0}$ contains all $P$-null sets); $\omega_{i}(t)$ stands for the independent standard Brownian motions defined on this probability space; $R_{+}^{n}$ is the positive cone in $R^{n}$, where has 
$R_{+}^{n}=\left\{x \in R^{n}: x_{i}>0\right.$ for all $\left.1 \leq i \leq n\right\} ; \tau>0$ and $C\left([-\tau, 0] ; R_{+}^{n}\right)$ denotes the family of continuous functions from $[-\tau, 0]$ to $R_{+}^{n}$.

\section{Model formulation and preliminaries}

The stochastic LV model with time delay studied in this paper can be given by

$$
\begin{aligned}
\mathrm{d} x_{i}(t)= & x_{i}(t)\left[r_{i}(t)-\sum_{j=1}^{n} a_{i j}(t) x_{j}(t)+\sum_{j=1}^{n} b_{i j}(t) x_{j}(t-\tau)+u_{i}(t)\right] \mathrm{d} t \\
& +\sigma_{i}(t) x_{i}(t) \mathrm{d} \omega_{i}(t)
\end{aligned}
$$

where $u_{i}(t), 1 \leq i \leq n$ denote the control input, $r_{i}(t), a_{i j}(t), b_{i j}(t), \sigma_{i}(t)$ are continuous bounded nonnegative functions on $[0, \infty)$.

Let the target model and the designed control law be

$$
\mathrm{d} y_{i}(t)=y_{i}(t)\left[r_{i}(t)-\sum_{j=1}^{n} a_{i j}(t) y_{j}(t)\right] \mathrm{d} t+\sigma_{i}(t) y_{i}(t) \mathrm{d} \omega_{i}(t), \quad i=1,2, \ldots, n,
$$

and

$$
u_{i}(t)=k_{i}\left(x_{i}(t)-y_{i}(t)\right)-\sum_{j=1}^{n} b_{i j} x_{j}(t-\tau)
$$

where $k_{i}$ is a constant.

Substituting (4) into (2), Eq. (3) becomes

$$
\begin{aligned}
\mathrm{d} x_{i}(t)= & x_{i}(t)\left[r_{i}(t)+k_{i}\left(x_{i}(t)-y_{i}(t)\right)-\sum_{j=1}^{n} a_{i j}(t) x_{j}(t)\right] \mathrm{d} t \\
& +\sigma_{i}(t) x_{i}(t) \mathrm{d} \omega_{i}(t) .
\end{aligned}
$$

The aim of this paper is to show that synchronization between model (2) and the target model (3) in Sect. 2 by designing effective controllers. Different from some existing results, we discuss the synchronization problem. When model (3) gets stochastic persistence, model (2) will also reach stochastic permanence. These results may certainly help to protect endangered animals and plants. As another innovation, we give a control variable, which precisely provides optimal control strategies.

With the help of Itô's formula, calculating the stochastic derivative of $\mathrm{d}\left(\log x_{i}(t)\right)$ to $(5)$, one has

$$
\begin{aligned}
\mathrm{d}\left(\log x_{i}(t)\right)= & {\left[r_{i}(t)-\frac{1}{2} \sigma_{i}^{2}(t)+k_{i}\left(x_{i}(t)-y_{i}(t)\right)-\sum_{j=1}^{n} a_{i j}(t) x_{j}(t)\right] \mathrm{d} t } \\
& +\sigma_{i}(t) \mathrm{d} \omega_{i}(t) .
\end{aligned}
$$


Similarly, apply Itô's formula to (3)

$$
\begin{aligned}
\mathrm{d}\left(\log y_{i}(t)\right)= & {\left[r_{i}(t)-\frac{1}{2} \sigma_{i}^{2}(t)-\sum_{j=1}^{n} a_{i j}(t) y_{j}(t)\right] \mathrm{d} t } \\
& +\sigma_{i}(t) \mathrm{d} \omega_{i}(t), \quad i=1,2, \ldots, n .
\end{aligned}
$$

Then

$$
\mathrm{d}\left(\log x_{i}(t)-\log y_{i}(t)\right)=\left[k_{i}\left(x_{i}(t)-y_{i}(t)\right)-\sum_{j=1}^{n} a_{i j}(t)\left(x_{j}(t)-y_{j}(t)\right)\right] \mathrm{d} t .
$$

Throughout this paper, the following assumptions and lemmas will be used.

Assumption 2.1 For $i, j=1,2, \ldots, n, \inf _{t \in[0,+\infty)} a_{i i}(t)>0, \inf _{t \in[0,+\infty)} a_{i j}(t) \geq 0$.

Assumption 2.2 For $i, j=1,2, \ldots, n$, there exist some constants $c_{i}, d_{i}, \lambda_{i j}, \rho_{i j} \geq 0, \lambda_{i i}>0$, and $\rho_{i i}>0$ such that

$$
d_{i}-\sum_{j=1}^{n} \rho_{i j} x_{j}(t) \leq r_{i}(t)-\sum_{j=1}^{n} a_{i j}(t) x_{j}(t)+\sum_{j=1}^{n} b_{i j}(t) x_{j}(t-\tau)+u_{i}(t) \leq c_{i}-\sum_{j=1}^{n} \lambda_{i j} x_{j}(t) .
$$

Assumption $2.3 \inf _{t \in[0,+\infty)}\left(a_{i i}(t)-k_{i}-\sum_{j=1, j \neq i}^{n} a_{j i}(t)\right)>0$ for all $1 \leq i \leq n$.

Lemma 2.1 Under Assumptions 2.1 and 2.2, for any given initial value $\{x(t):-\tau \leq t \leq$ $0\} \in C\left([-\tau, 0] ; R_{+}^{n}\right)$, there is a unique solution $x(t)$ to model $(2)$, which will remain in $R_{+}^{n}$ with probability 1 , namely $x(t) \in R_{+}^{n}$ for all $t \geq-\tau$ almost surely (a.s.).

Proof Since the coefficients of the equation are locally Lipschitz continuous, for any given initial value $\{x(t):-\tau \leq t \leq 0\} \in C\left([-\tau, 0] ; R_{+}^{n}\right)$ there is a unique maximal local solution $x(t)$ on $t \in\left[-\tau, \tau_{e}\right]$, where $\tau_{e}$ is the explosion time. To show this solution is global, we need to show that $\tau_{e}=\infty$. Let $k_{0}>0$ be sufficiently large for

$$
\frac{1}{k_{0}}<\min _{-\tau \leq t \leq 0}|x(t)| \leq \max _{-\tau \leq t \leq 0}|x(t)|<k_{0}
$$

For each integer $k \geq k_{0}$, define the stopping time $\tau_{k}=\inf \left\{t \in\left[0, \tau_{e}\right): x_{i}(t) \notin\left(\frac{1}{k}, k\right)\right.$ for some $i$. For simplicity, let inf $\phi=\infty$ ( $\phi$ denotes the empty set), the sequence $\tau_{k}$ is monotonically increasing. Let $\tau_{\infty}:=\lim _{k \rightarrow \infty} \tau_{k}, \tau_{\infty} \leq \tau_{e}$. It suffices to show that $\tau_{\infty}=\infty$ a.s. If this were false, there would exist $T>0$ and $\varepsilon>0$ such that $P\left\{\tau_{\infty} \leq T\right\}>\varepsilon$. Therefore we could find some $k_{1} \geq k_{0}$ such that $P\left\{\tau_{k} \leq T\right\}>\varepsilon$ for all $k \geq k_{1}$. Consider the Lyapunov function

$$
V(x(t))=\sum_{i=1}^{n}\left(x_{i}(t)-1-\log x_{i}(t)\right)
$$


Then $V(x(t)) \geq 0$ for every $x(t) \in R_{+}^{n}$. Apply the Itô formula to $V(x(t))$ to obtain

$$
\begin{aligned}
\mathcal{L} V(x(t))= & \sum_{i=1}^{n}\left(x_{i}(t)-1\right)\left[r_{i}(t)-\sum_{j=1}^{n} a_{i j}(t) x_{j}(t)+\sum_{j=1, j \neq i}^{n} b_{i j}(t) x_{j}(t-\tau)\right. \\
& \left.+u_{i}(t)\right]+\frac{1}{2} \sum_{i=1}^{n} \sigma_{i}^{2}(t) \\
\leq & \sum_{i=1}^{n} x_{i}(t)\left[c_{i}-\sum_{j=1}^{n} \lambda_{i j} x_{j}(t)\right]-\sum_{i=1}^{n}\left[d_{i}-\sum_{j=1}^{n} \rho_{i j} x_{j}(t)\right]+\frac{1}{2} \sum_{i=1}^{n} \sigma_{i}^{2}(t) \\
\leq & \sum_{i=1}^{n}\left[-\lambda_{i i} x_{i}^{2}(t)+\left(c_{i}+\sum_{j=1}^{n} \rho_{i j}\right) x_{j}(t)+\frac{1}{2} \max \left\{\sigma_{i}^{2}(t)\right\}-d_{i}\right] \\
\leq & K,
\end{aligned}
$$

where $K$ is a positive constant.

Based on the above analysis, note that, since $\lambda_{i i}>0$ for each $i$, the function $-\lambda_{i i} x_{i}^{2}(t)+$ $\left(c_{i}+\sum_{j=1}^{n} \rho_{i j}\right) x_{j}(t)+\frac{1}{2} \max \left\{\sigma_{i}^{2}(t)\right\}-d_{i}$ is continuous for $x_{i}(t) \in[0, \infty)$ and goes to $-\infty$ as $x_{i} \rightarrow \infty$. Therefore, we can obtain

$$
\int_{0}^{\tau_{k} \wedge T} \mathrm{~d} V(x(t)) \leq \int_{0}^{\tau_{k} \wedge T} K \mathrm{~d} t+\int_{0}^{\tau_{k} \wedge T} \sum_{i=1}^{n}\left(x_{i}(t)-1\right) \sigma_{i}(t) \mathrm{d} \omega_{i}(t) .
$$

Taking the mathematical expectation of both sides of (10) yields

$$
E V\left(x\left(\tau_{k} \wedge T\right)\right) \leq V(x(0))+K E\left(\tau_{k} \wedge T\right) \leq V(x(0))+K T
$$

It follows that

$$
V(x(0))+K T \geq E V\left(x\left(\tau_{k} \wedge T\right)\right) \geq E V\left[x\left(\tau_{k}\right) 1_{\left\{\tau_{k} \leq T\right\}}\right] .
$$

Note that $v \in\left\{\tau_{k} \leq T\right\}$. There are some $i$ such that $x_{i}\left(\tau_{k}(v)\right) \geq k$ or $x_{i}\left(\tau_{k}(\nu)\right) \leq \frac{1}{k}$, and hence $V\left(x\left(\tau_{k}, v\right)\right)$ is less than $k-1-\log (k)$ or $\frac{1}{k}-1-\log \left(\frac{1}{k}\right)=\frac{1}{k}-1+\log (k)$. Consequently,

$$
V\left(x\left(\tau_{k}, v\right)\right) \geq[k-1-\log (k)] \wedge\left[\frac{1}{k}-1+\log (k)\right] .
$$

Combining (11) with (12), the following inequality holds:

$$
V(x(0))+K T \geq \varepsilon\left([k-1-\log (k)] \wedge\left[\frac{1}{k}-1+\log (k)\right]\right) .
$$

This leads to a contradiction as $k \rightarrow \infty$. Therefore, $\tau_{\infty}=\infty$ a.s.

Lemma 2.2 ([12]) For any given initial value $y(0) \in R_{+}^{n}$, there is a unique solution $y(t)$ to model (3) on $t \geq 0$ and the solution will remain in $R_{+}^{n}$ with probability 1 , namely $y(t) \in R_{+}^{n}$ for all $t \geq 0$ a.s. 
Definition 2.1 The systems (2) and (3) are said to reach synchronization if, for any given initial conditions, the solutions of Eqs. (2) and (3) satisfy $\lim _{t \rightarrow \infty}\left|x_{i}(t)-y_{i}(t)\right|=0$, for all $1 \leq i \leq n$.

Definition 2.2 The systems (2) and (3) are said to reach partial synchronization if, for any given initial conditions, the solutions of systems (2) and (3) satisfy $\lim _{t \rightarrow \infty}\left|x_{i}(t)-y_{i}(t)\right|=0$, for all $1 \leq i \leq l<n$.

Consider the $n$-dimensional stochastic differential equation

$$
\mathrm{d} x(t)=f(x(t), t) \mathrm{d} t+g(x(t), t) \mathrm{d} B(t),
$$

where $B(t)=\left(B_{1}(t), B_{2}(t), \ldots, B_{n}(t)\right), f: R^{n} \times \bar{R}_{+} \rightarrow R^{n}, g: R^{n} \times \bar{R}_{+} \rightarrow R^{n \times m}$ and $\bar{R}_{+}=\{x(t) \in$ $R: x_{i}(t) \geq 0$ for $\left.1 \leq i \leq n\right\}$.

To proceed with the analysis, the nonlinear functions are assumed to satisfy the condition given below, which is presented in [9]:

(A) For any initial value, Eq. (13) has a unique solution. Moreover, for every $h>0$, there is a $K_{h}>0$ such that $|f(x(t), t)| \vee|g(x(t), t)| \leq K_{h}$, for all $t \geq 0$ and $x \in R^{n}$ with $|x(t)| \leq h$.

Lemma 2.3 ([12]) Let condition (A) hold. Assume that there exist three functions $V(t) \in$ $C^{2,1}\left(R^{n} \times \bar{R}_{+} ; \bar{R}_{+}\right), \gamma(t) \in L^{1}\left(\bar{R}_{+} ; \bar{R}_{+}\right)$, and $\varphi(t) \in C\left(R^{n} ; \bar{R}_{+}\right)$such that

$$
L V(x(t), t) \leq \gamma(t)-\varphi(x(t)), \quad(x(t), t) \in R^{n} \times \bar{R}_{+},
$$

and

$$
\lim _{|x(t)| \rightarrow \infty} \inf _{t \in[0,+\infty)} V(x(t), t)=\infty .
$$

Then $\operatorname{Ker}(\varphi) \neq \phi$ and $\lim _{t \rightarrow \infty} d(x(t, x(0)), \operatorname{Ker}(\varphi))=0$ a.s. for every $x(0) \in R^{n}$.

\section{Main results}

In this section, we derive some synchronization schemes by using the full controller and pinning controller, respectively.

Theorem 3.1 Under Assumption 2.3 and control law (4), for any given initial conditions, system (2) synchronizes with the target model (3).

Proof The proof is divided into two steps.

Step 1. According to Eq. (7), we can obtain

$$
\mathrm{d}\left(\log x_{i}(t)-\log y_{i}(t)\right)=\left[k_{i}\left(x_{i}(t)-y_{i}(t)\right)-\sum_{j=1}^{n} a_{i j}(t)\left(x_{j}(t)-y_{j}(t)\right)\right] \mathrm{d} t .
$$

Consider the Lyapunov function $\bar{V}(t)$ defined by

$$
\bar{V}(t)=\sum_{i=1}^{n}\left|\log x_{i}(t)-\log y_{i}(t)\right|
$$


A direct differential of $\bar{V}(t)$ along the ordinary differential equation (8) leads to

$$
\begin{aligned}
\mathrm{d} \bar{V}(t) & =\sum_{i=1}^{n} \operatorname{sgn}\left(x_{i}(t)-y_{i}(t)\right) \mathrm{d}\left(\log x_{i}(t)-\log y_{i}(t)\right) \\
& =-\sum_{i=1}^{n} \operatorname{sgn}\left(x_{i}(t)-y_{i}(t)\right)\left[\sum_{j=1}^{n} a_{i j}(t)\left(x_{j}(t)-y_{j}(t)\right)-k_{i}\left(x_{i}(t)-y_{i}(t)\right)\right] \mathrm{d} t \\
& \leq-\sum_{i=1}^{n}\left(a_{i i}(t)-k_{i}\right)\left|x_{i}(t)-y_{i}(t)\right| \mathrm{d} t+\sum_{i=1}^{n} \sum_{j=1, j \neq i}^{n} a_{i j}(t)\left|x_{i}(t)-y_{i}(t)\right| \mathrm{d} t \\
& =-\sum_{i=1}^{n}\left(a_{i i}(t)-k_{i}\right)\left|x_{i}(t)-y_{i}(t)\right| \mathrm{d} t+\sum_{i=1}^{n} \sum_{j=1, j \neq i}^{n} a_{j i}(t)\left|x_{i}(t)-y_{i}(t)\right| \mathrm{d} t \\
& \leq-\min _{1 \leq i \leq n} \inf _{t \in[0,+\infty)}\left(a_{i i}(t)-k_{i}-\sum_{j=1, j \neq i}^{n} a_{j i}(t)\right)\left|x_{i}(t)-y_{i}(t)\right| \mathrm{d} t \\
& =-\varpi \sum_{i=1}^{n}\left|x_{i}(t)-y_{i}(t)\right| \mathrm{d} t,
\end{aligned}
$$

where $\varpi=\min _{1 \leq i \leq n} \inf _{t \in[0,+\infty)}\left(a_{i i}(t)-k_{i}-\sum_{j=1, j \neq i}^{n} a_{j i}(t)\right)>0$.

Integrating from 0 to $t$ on both sides of (16), we have

$$
\bar{V}(t)+\varpi \int_{0}^{t} \sum_{i=1}^{n}\left|x_{i}(s)-y_{i}(s)\right| d s \leq \bar{V}(0)<\infty
$$

Let $t \rightarrow \infty$, the following inequality is obtained:

$$
\int_{0}^{t}|x(s)-y(s)| d s \leq \int_{0}^{t} \sum_{i=1}^{n}\left|x_{i}(s)-y_{i}(s)\right| d s \leq \frac{\bar{V}(0)}{\varpi}<\infty .
$$

Moreover, we also have

$$
E \int_{0}^{t}|x(s)-y(s)| d s<\infty
$$

Step 2. Define $e(t)=x(t)-y(t)$. Clearly, $e(t) \in C\left(R_{+}, R\right)$. It is straightforward to obtain from (16)

$$
\lim _{t \rightarrow \infty} \inf |e(t)|=0, \quad \text { a.s. }
$$

Now we claim that $\lim _{t \rightarrow \infty}|e(t)|=0$ a.s.

The following proof is similar to Theorem 6.2 in [12], so it is omitted.

Remark 1 Generally speaking, stochastic permanence of the population system has certainly significance for the endangered treasure species, which has stirred great research attention [26-30]. However, this is the first time to present some theoretical results on the asymptotically synchronization for the stochastic LV model. Moreover, in addition, the proposed results can be viewed as an extension of those in [31-35]. 
To study the synchronization problem of extinction, let us impose one more hypothesis.

Assumption $3.1 \sum_{i=1}^{n} \int_{0}^{\infty}\left[r_{i}(t)-\frac{1}{2}\left(1-k_{i}\right) \sigma_{i}^{2}(t)\right]^{+} \mathrm{d} t<\infty$ for all $0<k_{i} \ll 1, i=1,2, \ldots, n$.

Corollary 3.1 Under Assumptions 2.3 and 3.1, for any given initial value, if the population becomes extinct in the target model (3), then the solution $x_{i}(t)$ of model (5) has the property

$$
\lim _{t \rightarrow \infty}\left|x_{i}(t)\right|=0 \quad \text { a.s. }
$$

Proof It is obvious that model (5) satisfies (A). By the Itô formula, one has

$$
\begin{aligned}
\mathrm{d} x_{i}^{k_{i}}(t)= & k_{i} x_{i}^{k_{i}}(t)\left[r_{i}(t)+k_{i} x_{i}(t)-\sum_{j=1}^{n} a_{i j}(t) x_{j}(t)+\frac{\left(k_{i}-1\right) \sigma_{i}^{2}(t)}{2}\right] \mathrm{d} t \\
& +k_{i} x_{i}^{k_{i}}(t) \sigma_{i}(t) \mathrm{d} B_{i}(t) \\
= & k_{i} x_{i}^{k_{i}}(t)\left(r_{i}(t)-\frac{\left(1-k_{i}\right) \sigma_{i}^{2}(t)}{2}\right) \mathrm{d} t+k_{i}^{2} x_{i}^{k_{i}+1}(t) \mathrm{d} t \\
& -k_{i} x_{i}^{k_{i}}(t) \sum_{j=1}^{n} a_{i j}(t) x_{j}(t) \mathrm{d} t+k_{i} x_{i}^{k_{i}}(t) \sigma_{i}(t) \mathrm{d} \omega_{i}(t) \\
\leq & k_{i} x_{i}^{k_{i}}(t)\left(r_{i}(t)-\frac{\left(1-k_{i}\right) \sigma_{i}^{2}(t)}{2}\right) \mathrm{d} t+k_{i}\left(k_{i}-a_{i i}(t)\right) x_{i}^{k_{i}+1}(t) \mathrm{d} t \\
& +k_{i} x_{i}^{k_{i}}(t) \sigma_{i}(t) \mathrm{d} \omega_{i}(t) .
\end{aligned}
$$

Define $W_{i}(t)=e^{-k_{i} \int_{0}^{t}\left(r_{i}(s)-\frac{\left(1-k_{i}\right) \sigma_{i}^{2}(s)}{2}\right) d s}$ and $\widetilde{V}(x(t), t)=\sum_{i=1}^{n} W_{i}(t) x_{i}^{k_{i}}(t)$.

Under Assumption 3.1, $\widetilde{V} \in C^{2,1}\left(R^{n} \times \bar{R}_{+} ; \bar{R}_{+}\right)$and $\lim _{|x| \rightarrow \infty} \inf _{t \in[0,+\infty)} V(x, t)=\infty$. There exists a positive constant $Q_{i}$ such as $W_{i}(t) \geq Q_{i}$. As it follows from Itô’s formula, we have

$$
\begin{aligned}
\mathrm{d} \widetilde{V}(x(t), t)= & -\sum_{i=1}^{n} k_{i} W_{i}(t) x_{i}^{k_{i}}(t)\left(r_{i}(t)-\frac{\left(1-k_{i}\right) \sigma_{i}^{2}(t)}{2}\right) \mathrm{d} t+\sum_{i=1}^{n} W_{i}(t) \mathrm{d} x_{i}^{k_{i}}(t) \\
\leq & -\sum_{i=1}^{n} k_{i}\left(a_{i i}-k_{i}\right) W_{i}(t) x_{i}^{k_{i}+1}(t) \mathrm{d} t+\sum_{i=1}^{n} k_{i} W_{i}(t) x_{i}^{k_{i}}(t) \sigma_{i}(t) \mathrm{d} \omega_{i}(t) \\
\leq & -\sum_{i=1}^{n}\left[\inf _{t \in[0,+\infty)}\left(a_{i i}-k_{i}\right)\right] k_{i} Q_{i} x_{i}^{k_{i}+1}(t) \mathrm{d} t \\
& +\sum_{i=1}^{n} k_{i} W_{i}(t) x_{i}^{k_{i}}(t) \sigma_{i}(t) \mathrm{d} \omega_{i}(t) \\
= & -\sum_{i=1}^{n} \delta_{i} k_{i} Q_{i} x_{i}^{k_{i}+1}(t) \mathrm{d} t+\sum_{i=1}^{n} k_{i} W_{i}(t) x_{i}^{k_{i}}(t) \sigma_{i}(t) \mathrm{d} \omega_{i}(t),
\end{aligned}
$$

where $\delta_{i}=\inf _{t \in[0,+\infty)}\left(a_{i i}-k_{i}\right)>0$. Under Assumption 2.3, it is easy to have, therefore,

$$
L \widetilde{V}(x(t), t) \leq-\sum_{i=1}^{n} k_{i} \delta_{i} Q_{i} x_{i}^{k_{i}+1}(t)=:-\omega(x(t)) .
$$


With the help of Lemma 2.3, (20) is obtained.

To achieve partial synchronization, the control input $u_{i}(t)$ can be designed as

$$
\left\{\begin{array}{l}
u_{i}(t)=k_{i}\left(x_{i}(t)-y_{i}(t)\right)-\sum_{j=1}^{n} b_{i j} x_{j}(t-\tau), \quad i=1,2, \ldots, l, \\
u_{i}(t)=0, \quad i=l+1, l+2, \ldots, n .
\end{array}\right.
$$

Accordingly, the model (2) can be rewritten in the following form:

$$
\left\{\begin{aligned}
\mathrm{d} \log x_{i}(t)= & {\left[r_{i}(t)-\frac{1}{2} \sigma_{i}^{2}(t)+k_{i}\left(x_{i}(t)-y_{i}(t)\right)-\sum_{j=1}^{n} a_{i j}(t) x_{j}(t)\right] \mathrm{d} t } \\
& +\sigma_{i}(t) \mathrm{d} \omega_{i}(t), \quad i=1,2, \ldots, l \\
\mathrm{~d} \log x_{i}(t)= & {\left[r_{i}(t)-\frac{1}{2} \sigma_{i}^{2}(t)-\sum_{j=1}^{n} a_{i j}(t) x_{j}(t-\tau)\right] \mathrm{d} t } \\
& +\sigma_{i}(t) \mathrm{d} \omega_{i}(t), \quad i=l+1, l+2, \ldots, n .
\end{aligned}\right.
$$

For Eq. (24), it is difficult to achieve synchronous control. At this point, we can only guarantee partial synchronization, and Assumption 2.3 is modified as

$$
\inf _{t \in[0,+\infty)}\left(a_{i i}(t)-k_{i}-\sum_{j=1, j \neq i}^{n} a_{j i}(t)\right)>0, \quad 1 \leq i \leq l .
$$

Then, using the same idea as in Theorem 3.1, we obtain the following theorem.

Theorem 3.2 Under (25) and control law (23), for any given initial conditions, model (2) partially synchronizes the target model (3).

Remark 2 It is natural to consider the pinning control method to realize synchronization of systems (3) and (24) via control of partial species instead of all species, which can effectively reduce control cost, especially in limited energy systems. In addition, it shows practical significance.

\section{An illustrative example}

In order to verify the effectiveness of the theoretical results, we will present one numerical example in this section. Consider the stochastic model (2) with three populations $(n=3)$, where the system is described by

$$
a_{i j}(t)=\left(\begin{array}{ccc}
3 t^{5} & 4 t & 4 t \\
4 t & 4 t^{5} & 5 t \\
2 t & 2 t & 5 t^{5}
\end{array}\right), \quad b_{i j}(t)=\left(\begin{array}{ccc}
3 t^{5} & 2 t & t \\
t & 2 t^{5} & t \\
t & t & 3 t^{5}
\end{array}\right)
$$

with $r(t)=\left(3 t^{5}, 4 t^{5}, 4 t^{5}\right), \sigma(t)=\left(0.3 t^{\frac{1}{5}}, 0.4 t^{\frac{1}{5}}, 0.2 t^{\frac{1}{5}}\right), \tau=0.3$, and initial state $x(t)=$ $\left(e^{-1.5}, e^{-0.6}, e^{-1}\right), y(t)=\left(e^{-1}, e^{-0.5}, e^{-0.8}\right)$.

It can be checked that Assumptions 2.1-2.3 and 3.1 are satisfied with $d_{i}=-2, c_{i}=8 T^{5}+$ $3 T(i=1,2,3), k=(0.007,0.006,0.017)$,

$$
\rho_{i j}=\left(\begin{array}{ccc}
3 T^{5} & 4 T & 4 T \\
4 T & 4 T^{5} & 5 T \\
2 T & 2 T & 5 T^{5}
\end{array}\right), \quad \lambda_{i j}=\left(\begin{array}{ccc}
3 T^{5} & 0 & 0 \\
0 & 2 T^{5} & 0 \\
0 & 0 & 3 T^{5}
\end{array}\right) .
$$



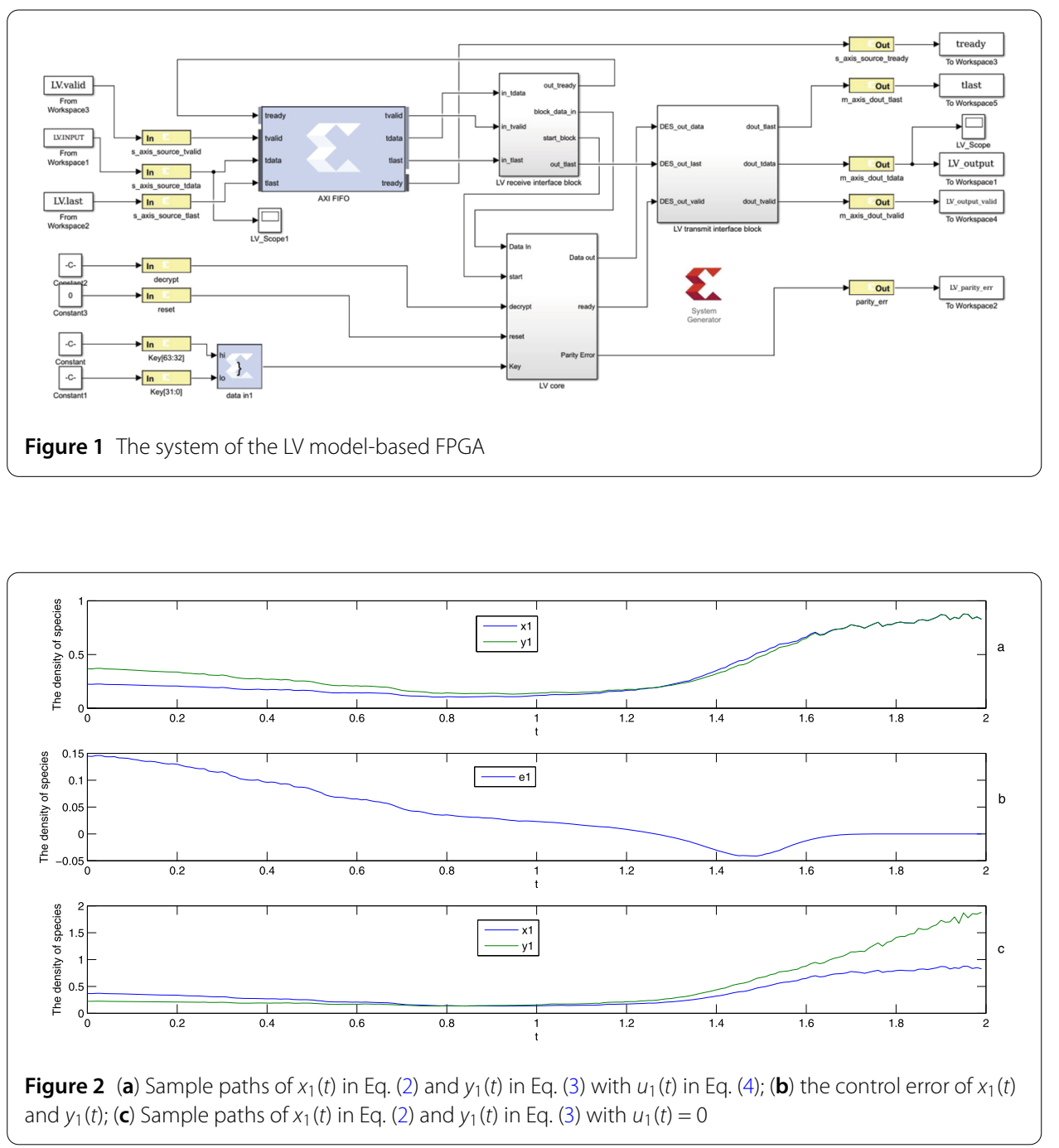

Based on the current mainstream Xilinx ZYNQ series of FPGA chips, we have built our system in a hardware way. The hardware system diagram is shown in Fig. 1. The input and output data signals of the module are named in the format which is compatible with AXIStream Interface specifications. Therefore, this module is capable of being generated and selected as an IP core in Vivado, and supporting AXI-Stream interface. Besides the data IOs, all other IOs are in the format of the AXI-Lite interface. As shown in Fig. 1, constant parameter matrices are transmitted to the $\mathbf{L V}$ _core through data in 1 module. At the same time, input data from work spaces are transmitted to the LV_core through AXI FIFO and $\mathrm{LV}$ receive interface block. The $L V$ _core will calculate the result, and then the calculated result will be outputted through the $\mathbf{L V}$ transmit interface block.

Figures 2(a), (b), 3(a), (b), and 4(a), (b) show that the three populations have good synchronization under control. Figures 2(c), 2(c), and 4(c) show that the three populations cannot synchronize without control. These figures imply that the three populations partially synchronize with pinning control.

The hardware resource consumption of the system is shown in Table 1. 

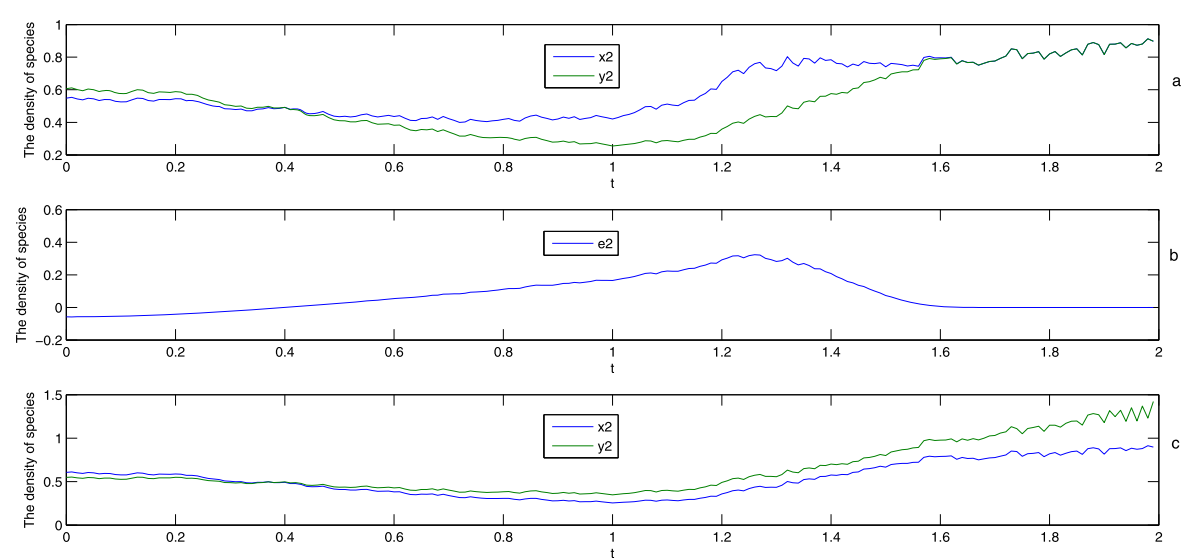

Figure 3 (a) Sample paths of $x_{2}(t)$ in Eq. (2) and $y_{2}(t)$ in Eq. (3) with $u_{2}(t)$ in Eq. (4); (b) the control error of $x_{2}(t)$ and $y_{2}(t)$; (c) Sample paths of $x_{2}(t)$ in Eq. (2) and $y_{2}(t)$ in Eq. (3) with $u_{2}(t)=0$
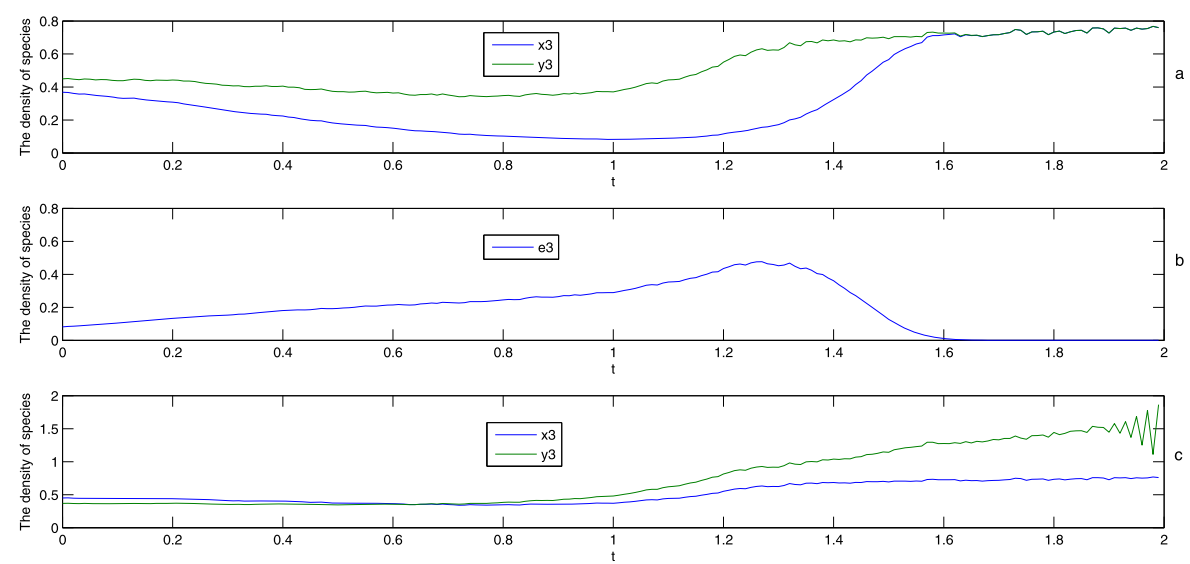

Figure 4 (a) Sample paths of $x_{3}(t)$ in Eq. (2) and $y_{3}(t)$ in Eq. (3) with $u_{3}(t)$ in Eq. (4); (b) the control error of $x_{3}(t)$ and $y_{3}(t) ;(\mathbf{c})$ Sample paths of $x_{3}(t)$ in Eq. (2) and $y_{3}(t)$ in Eq. (3) with $u_{3}(t)=0$

Table 1 Results for resource consumption

\begin{tabular}{lcrl}
\hline Resource & Utilization & Available & Utilization \\
\hline LUT & 1061 & 53,200 & 1.994 \\
LUTRAM & 65 & 17,400 & 0.374 \\
FF & 1334 & 106,400 & 1.254 \\
BRAM & 1.5 & 140 & 1.071 \\
BUFG & 1 & 32 & 3.125 \\
\hline
\end{tabular}

\section{Conclusions}

In this paper, the synchronization control problem for a non-autonomous LV system with time delay and stochastic effects was analyzed by the stochastic technique and Lyapunov stability theory. The full controller and pinning controller have been designed, respectively. Synchronous control of population is meant to achieve the expected extent of the population. This has certainly significance for the endangered treasure species. The proposed model can achieve stochastically permanent synchronization. Further, the synchronization problem of extinction was studied. Moreover, through the FPGA hardware tool, 
the speed of simulation processing was improved to meet the needs of the real-time world with massive data in the era of big data. This will be a challenging task in the next work.

\author{
Acknowledgements \\ The authors would like to thank the editor and the anonymous reviewers for their valuable comments and constructive \\ suggestions.
}

\title{
Funding
}

This work was jointly supported by Academic Leader High-end Research and Study Project for Jiangsu Higher Vocational Colleges Teachers of China (No. 2019GRGDYX129), Natural Science Research of Jiangsu Higher Education Institutions of China (No. 19KJB120013), Qinglan Project of Jiangsu Province of China (No. 014000773/2018-00376), Program for International Science and Technology Cooperation Projects of Jiangsu Province of China (No. BZ2018031) and Program for International Science and Technology Cooperation Projects of Wuxi City of China (No. WX0303B010518180009PB).

\section{Availability of data and materials}

Data sharing is not applicable to this article as no data sets were generated or analyzed during the current study.

\section{Competing interests}

The authors declare that they have no competing interests.

\section{Authors' contributions}

All authors read and approved the final manuscript.

\section{Author details}

${ }^{1}$ Department of Fundamental Courses, Wuxi Insitute of Technology, Wuxi, P.R. China. ${ }^{2}$ School of Mathematics, Southeast University, Nanjing, P.R. China. ${ }^{3}$ China Key System \& Integrated Circuit Co., Ldt., Wuxi, P.R. China. ${ }^{4}$ School of Life Sciences, Sun Yat-sen University, Guangzhou, P.R. China.

\section{Publisher's Note}

Springer Nature remains neutral with regard to jurisdictional claims in published maps and institutional affiliations.

Received: 4 October 2019 Accepted: 18 December 2019 Published online: 03 January 2020

\section{References}

1. Freedman, H.I.: Deterministic Mathematical Models in Population Ecology. Dekker, New York (1980)

2. Tahara, T., Gavina, M.K.A., Kawano, T., et al.: Asymptotic stability of a modified Lotka-Volterra model with small immigrations. Sci. Rep. 8(1), 7029 (2018)

3. Ma, L., Guo, S.: Stability and bifurcation in a diffusive Lotka-Volterra system with delay. Comput. Math. Appl. 72(1) 147-177 (2016)

4. Wang, X., Du, Z., Liang, J.: Existence and global attractivity of positive periodic solution to a Lotka-Volterra model. Nonlinear Anal., Real World Appl. 11(5), 4054-4061 (2010)

5. Vaidyanathan, S.: Adaptive synchronization of generalized Lotka-Volterra three-species biological systems. Int. J. PharmTech Res. 8(5), 928-937 (2015)

6. He, X., Ni, W.M.: Global dynamics of the Lotka-Volterra competition-diffusion system: diffusion and spatial heterogeneity I. Commun. Pure Appl. Math. 69(5), 981-1014 (2016)

7. Meng, X., Zhang, L.: Evolutionary dynamics in a Lotka-Volterra competition model with impulsive periodic disturbance. Math. Methods Appl. Sci. 39(2), 177-188 (2016)

8. Cao, J., Guerrini, L., Cheng, Z.: Stability and Hopf bifurcation of controlled complex networks model with two delays Appl. Math. Comput. 343, 21-29 (2019)

9. Cao, Y.: Bifurcations in an Internet congestion control system with distributed delay. Appl. Math. Comput. 347, 54-63 (2019)

10. Li, Q., Liang, J., Gong, W.: Stability and synchronization for impulsive Markovian switching CVNNs: matrix measure approach. Commun. Nonlinear Sci. Numer. Simul. 77, 126-140 (2019)

11. Bahar, A., Mao, X.: Stochastic delay population dynamics. Int. J. Pure Appl. Math. 11, 377-400 (2004)

12. Li, X., Mao, X.: Population dynamical behavior of non-autonomous Lotka-Volterra competitive system with random perturbation. Discrete Contin. Dyn. Syst., Ser. A 24(2), 523-593 (2009)

13. Liu, M., Wang, K.: Persistence and extinction in stochastic non-autonomous logistic systems. J. Math. Anal. Appl. 375 443-457 (2011)

14. Choo, S.M., Kim, Y.H.: Global stability in n-dimensional stochastic difference equations for predator-prey models. J. Comput. Anal. Appl. 24(1), 136 (2018) 116

15. Shi, C., Li, Z., Chen, F.: Extinction in a nonautonomous Lotka-Volterra competitive system with infinite delay and feedback controls. Nonlinear Anal., Real World Appl. 13(5), 2214-2226 (2012)

16. Liu, M., Wang, K.: Stochastic Lotka-Volterra systems with Lévy noise. J. Math. Anal. Appl. 410(2), 750-763 (2014)

17. Wang, S., Hu, G., Wang, L.: Stability in distribution of a stochastic competitive Lotka-Volterra system with S-type distributed time delays. Methodol. Comput. Appl. Probab. 20(4), 1241-1257 (2018)

18. Lu, C., Ding, X.: Persistence and extinction of an impulsive stochastic logistic model with infinite delay. Osaka J. Math. 53(1), 1-31 (2016)

19. Liu, Q., Jiang, D., Hayat, T., et al.: Dynamics of a stochastic predator-prey model with distributed delay and Markovian switching. Physica A 527, 121264 (2019) 
20. Elsadany, A.A., Matouk, A.E., Abdelwahab, A.G., et al.: Dynamical analysis, linear feedback control and synchronization of a generalized Lotka-Volterra system. Int. J. Dyn. Control 6(1), 328-338 (2018)

21. Saad, A.B., Boubaker, O.: Bifurcations, chaos and synchronization of a predator-prey system with Allee effect and seasonally forcing in prey's growth rate. Eur. Phys. J. Spec. Top. 227(7-9), 971-981 (2018)

22. Lu, J., Kurths, J., Cao, J., et al.: Synchronization control for nonlinear stochastic dynamical networks: pinning impulsive strategy. IEEE Trans. Neural Netw. Learn. Syst. 23(2), 285-292 (2015)

23. Guan, Z.H., Yue, D., Hu, B., et al.: Cluster synchronization of coupled genetic regulatory networks with delays via aperiodically adaptive intermittent control. IEEE Trans. Nanobiosci. 16(7), 585-599 (2017)

24. Shi, L., Zhu, H., Zhong, S., et al.: Synchronization for time-varying complex networks based on control. J. Comput. Appl. Math. 301, 178-187 (2016)

25. Peng, H., Wei, N., Li, L., et al.: Models and synchronization of time-delayed complex dynamical networks with multi-links based on adaptive control. Phys. Lett. A 374, 2335-2339 (2010)

26. Lian, B., Hu, S.: Asymptotic behaviour of the stochastic Gilpin-Ayala competition models. J. Math. Anal. Appl. 339(1), 419-428 (2008)

27. Li, Q., Zhang, Q., Cao, B.: Mean-square stability of stochastic age-dependent delay population systems with jumps. Acta Math. Appl. Sin. 34(1), 145-154 (2018)

28. Valenti, D., Fiasconaro, A., Spagnolo, B.: Stochastic resonance and noise delayed extinction in a model of two competing species. Physica A 331(3-4), 477-486 (2004)

29. Vasilova, M., Jovanović, M.: Stochastic Gilpin-Ayala competition model with infinite delay. Appl. Math. Comput. 217(10), 4944-4959 (2011)

30. Liu, M., Zhu, Y.: Stationary distribution and ergodicity of a stochastic hybrid competition model with Lévy jumps. Nonlinear Anal. Hybrid Syst. 30, 225-239 (2018)

31. Liu, M., Wang, K.: Global asymptotic stability of a stochastic Lotka-Volterra model with infinite delays. Commun. Nonlinear Sci. Numer. Simul. 17(8), 3115-3123 (2012)

32. Tran, K., Yin, G.: Optimal harvesting strategies for stochastic competitive Lotka-Volterra ecosystems. Automatica 55, 236-246 (2015)

33. Xiong, J., Li, X., Wang, H.: Global asymptotic stability of a Lotka-Volterra competition model with stochasticity in inter-specific competition. Appl. Math. Lett. 89, 58-63 (2019)

34. Wang, S., Hu, G., Wang, L.: Stability in distribution of a stochastic competitive Lotka-Volterra system with S-type distributed time delays. Methodol. Comput. Appl. Probab. 20(4), 1241-1257 (2018)

35. Liu, L., Shen, Y: New criteria on persistence in mean and extinction for stochastic competitive Lotka-Volterra systems with regime switching. J. Math. Anal. Appl. 430(1), 306-323 (2015)

\section{Submit your manuscript to a SpringerOpen ${ }^{\circ}$ journal and benefit from:}

- Convenient online submission

- Rigorous peer review

- Open access: articles freely available online

- High visibility within the field

- Retaining the copyright to your article

Submit your next manuscript at $\gg$ springeropen.com 